PROCEEDINGS OF THE

AMERICAN MATHEMATICAL SOCIETY

Volume 130, Number 11, Pages 3433-3437

S 0002-9939(02)06709-6

Article electronically published on May 29, 2002

\title{
AN INFINITE FAMILY OF SUMMATION IDENTITIES
}

\author{
S. F. KEATING
}

(Communicated by Dennis A. Hejhal)

\begin{abstract}
Theta functions have historically played a prominent role in number theory. One such role is the construction of modular forms. In this work, a generalized theta function is used to construct an infinite family of summation identities. Our results grew out of some observations noted during a presentation given by the author at the 1992 AMS-MAA-SIAM Joint Meetings in Baltimore.
\end{abstract}

\section{INTRODUCTION}

Theta functions have historically played a prominent role in number theory. One such role is the use of theta functions to construct modular forms. In this context, we now take the classical theta function 8

$$
\vartheta(z)=\sum_{m=-\infty}^{\infty} e^{i \pi m^{2} z}, \quad \operatorname{Im}(z)>0
$$

which is absolutely convergent for $\operatorname{Im}(z)>0$ and satisfies

$$
\vartheta(z+2)=\vartheta(z)
$$

and

$$
\vartheta\left(\frac{-1}{z}\right)=(\sqrt{-i z}) \vartheta(z) .
$$

The square root is defined to be positive on the positive imaginary axis. Equations (2) and (3) establish that $\vartheta(z)$ is a modular form of weight $1 / 2$ for the group $\mathrm{G}(2)$ generated by $z \rightarrow z+2$ and $z \rightarrow \frac{-1}{z}[9$.

Take $z=i t$ in (11) to obtain the Jacobi theta function

$$
\theta(t)=\sum_{m \in \mathbf{Z}} e^{-\pi m^{2} t} \quad \text { for } t>0 .
$$

$\theta(t)$ satisfies the Jacobi inversion formula

$$
\theta(t)=\frac{1}{\sqrt{t}} \theta\left(\frac{1}{t}\right), \quad t>0, \sqrt{t}>0 .
$$

Received by the editors May 29, 2001.

2000 Mathematics Subject Classification. Primary 11-XX.

(C)2002 American Mathematical Society 
Equation (5) yields the summation identity

$$
\frac{1}{s}+2 \sum_{m=1}^{\infty} \frac{1}{s+\pi m^{2}}=\frac{\sqrt{\pi}}{\sqrt{s}}+2 \frac{\sqrt{\pi}}{\sqrt{s}} \sum_{m=1}^{\infty} e^{-2 m \sqrt{\pi s}} \quad \text { for } \operatorname{Re}(s)>0 .
$$

We note that (6) is equivalent to the summation identity presented by Bellman in [1] and can be viewed as a partial fraction expansion of the hyperbolic cotangent function, $\operatorname{coth}(\sqrt{\pi s})$, for $\operatorname{Re}(s)>0$.

In [5] the Jacobi theta function was generalized by

$$
\begin{gathered}
\theta_{n, l}(t)=\sum_{m \in \mathbf{Z}} e^{-\pi m^{2} t} m^{l} H_{n}(\sqrt{2 \pi t} m) \quad \text { if } l \neq 0, \\
\theta_{n, 0}(t)=\sum_{m \in \mathbf{Z}} e^{-\pi m^{2} t} H_{n}(\sqrt{2 \pi t} m) .
\end{gathered}
$$

Equations (7) and (8) hold for $n=0,1,2, \ldots$ and $t>0 . H_{n}(x)$ is the $n$th Hermite polynomial. We remark that theta functions akin to $\theta_{n, 0}(t)$ occur in a variety of different contexts; cf., e.g., 7, pp. 428-429, 447], [10, pp. 1014, 1045], and [11].

By using these generalized theta functions, and expressing $H_{n}(x)$ as a series [6], one obtains a generalized Jacobi inversion formula [5]:

$$
\begin{aligned}
\theta_{n, l}(t)=\frac{(-1)^{\frac{(n-l)}{2}} n ! l !}{t^{\frac{(2 l+1)}{2}}} \sum_{k=0}^{\min (n, l)} \sum_{j=0}^{\left[\frac{(l-k)}{2}\right]}\left(\frac{(-1)^{n-k-j}(2)^{\frac{(k-4 j)}{2}} \pi^{\frac{(-k-2 j)}{2}}}{j ! k !(l-k-2 j) !(n-k) !}\right) \\
\times \frac{1}{t^{\frac{(-k-2 j)}{2}}} \theta_{n-k, l-k-2 j}\left(\frac{1}{t}\right)
\end{aligned}
$$

In (9) the symbol [ ] denotes the greatest integer function and the inversion formula holds for $t>0, n, l=0,1,2, \ldots, n+l$ is an even, nonnegative integer.

Our primary aim in this note is to announce some new summation identities which are constructed via (9). Although these summation identities can be developed quite independently of modular forms, it is useful to recall the actual connection between (1) as a theta function and as a modular form and (4).

\section{An infinite family of SUmmation identities}

We first define a constant $c_{q}$, for $q=0,1,2, \ldots$, as

$$
c_{q}=\frac{(-1)^{q}(2 q) !}{q !} .
$$

If we consider the generalized Jacobi inversion formula for the case where $n=$ $2 q, q=0,1,2, \ldots$, and $l=0$, we obtain

$$
\theta_{2 q, 0}(t)=\frac{(-1)^{q}}{t^{\frac{1}{2}}} \theta_{2 q, 0}\left(\frac{1}{t}\right)
$$

We note that for $q=0$ (11) is equal to (5). 
We express (11) as a series and then apply the Laplace transform, interchanging summation and integral. The formulas found in [2], [3] and [4] are utilized and some algebraic simplification is implemented. We obtain the following infinite family of summation identities:

$$
\begin{aligned}
\frac{1}{s} c_{q}+ & 2 c_{q} \sum_{m=1}^{\infty} \frac{1}{s+\pi m^{2}} \\
& +\sum_{m=1}^{\infty} \sum_{k=0}^{q-1} \frac{(-1)^{k}(2 q) ! 2^{(3 q-3 k+1)} \pi^{(q-k)} m^{(2 q-2 k)}}{k !(2 q-2 k) !} \frac{(q-k) !}{\left(s+\pi m^{2}\right)^{(q-k+1)}} \\
= & (-1)^{q} c_{q} \frac{\sqrt{\pi}}{\sqrt{s}}+2(-1)^{q} c_{q} \frac{\sqrt{\pi}}{\sqrt{s}} \sum_{m=1}^{\infty} e^{-2 m \sqrt{\pi s}} \\
& +\sum_{m=1}^{\infty} \sum_{k=0}^{q-1} \frac{2^{(3 q-3 k+1)}(2 q) ! \pi^{\left(\frac{q-k+1}{2}\right)} m^{(q-k)} e^{-2 m \sqrt{\pi s}}}{k !(2 q-2 k) !(-1)^{(-q-k)} s^{\frac{-(q-k-1)}{2}}} \\
& \times \sum_{r=0}^{q-k-1} \frac{(q-k-1+r) !}{r !(q-k-1-r) ! 2^{(2 r)} m^{r} \pi^{\frac{r}{2}} s^{\frac{r}{2}}},
\end{aligned}
$$

for $q=0,1,2, \ldots, \operatorname{Re}(s)>0$.

If we consider the case where $q=2 \mu$ is an even, nonnegative integer in (12), expanding (11) and implementing algebraic simplification, we obtain

$$
\begin{aligned}
\frac{(4 \mu) !}{(2 \mu) !} \frac{1}{s} & +2 \frac{(4 \mu) !}{(2 \mu) !} \sum_{m=1}^{\infty} \frac{1}{s+\pi m^{2}} \\
& +\sum_{m=1}^{\infty} \sum_{k=0}^{2 \mu-1} \frac{(-1)^{k}(4 \mu) ! 2^{(6 \mu-3 k+1)} \pi^{(2 \mu-k)} m^{(4 \mu-2 k)}(2 \mu-k) !}{k !(4 \mu-2 k) !\left(s+\pi m^{2}\right)^{(2 \mu-k+1)}} \\
= & \frac{(4 \mu) !}{(2 \mu) !} \frac{\sqrt{\pi}}{\sqrt{s}}+2 \frac{(4 \mu) !}{(2 \mu) !} \frac{\sqrt{\pi}}{\sqrt{s}} \sum_{m=1}^{\infty} e^{-2 m \sqrt{\pi s}} \\
& +\sum_{m=1}^{\infty} \sum_{k=0}^{2 \mu-1} \frac{(-1)^{k} 2^{(6 \mu-3 k+1)}(4 \mu) ! \pi^{\left(\frac{2 \mu-k+1)}{2}\right)} m^{(2 \mu-k)} e^{-2 m \sqrt{\pi s}}}{k !(4 \mu-2 k) ! s^{\frac{-(2 \mu-k-1)}{2}}} \\
& \times \sum_{r=0}^{2 \mu-k-1} \frac{(2 \mu-k-1+r) !}{r !(2 \mu-k-1-r) ! 2^{(2 r)} m^{r} \pi^{\frac{r}{2}} s^{\frac{r}{2}}},
\end{aligned}
$$

for $\mu=0,1,2, \ldots, \operatorname{Re}(s)>0$.

As one might expect, when producing identities in the manner described, consideration of $l$ equal to a positive, even integer in (9) is similar but more cumbersome. 


\section{Some EXAmples}

The value $\mu=0$ applied to (13) yields the identity noted in (하). The value $\mu=1$ applied to (13) yields the following identity:

$$
\begin{aligned}
\frac{12}{s}+ & 24 \sum_{m=1}^{\infty} \frac{1}{s+\pi m^{2}}+\sum_{m=1}^{\infty} \frac{256 \pi^{2} m^{4}}{\left(s+\pi m^{2}\right)^{3}}-\sum_{m=1}^{\infty} \frac{192 \pi m^{2}}{\left(s+\pi m^{2}\right)^{2}} \\
= & \frac{12 \sqrt{\pi}}{\sqrt{s}}+\frac{24 \sqrt{\pi}}{\sqrt{s}} \sum_{m=1}^{\infty} e^{-2 m \sqrt{\pi s}}+\sum_{m=1}^{\infty} 128 \pi^{\frac{3}{2}} m^{2} s^{\frac{1}{2}} e^{-2 m \sqrt{\pi s}} \\
& -\sum_{m=1}^{\infty} 64 \pi m e^{-2 m \sqrt{\pi s}}
\end{aligned}
$$

Subtracting identity (6) from identity (14) and canceling common factors results in yet another identity:

$$
\begin{aligned}
\sum_{m=1}^{\infty} \frac{256 \pi^{2} m^{4}}{\left(s+\pi m^{2}\right)^{3}}-\sum_{m=1}^{\infty} \frac{192 \pi m^{2}}{\left(s+\pi m^{2}\right)^{2}}+\sum_{m=1}^{\infty} \frac{22}{s+\pi m^{2}}+\frac{11}{s} \\
=\sum_{m=1}^{\infty} 128 \pi^{\frac{3}{2}} m^{2} s^{\frac{1}{2}} e^{-2 m \sqrt{\pi s}}-\sum_{m=1}^{\infty} 64 \pi m e^{-2 m \sqrt{\pi s}} \\
\quad+22 \frac{\sqrt{\pi}}{\sqrt{s}} \sum_{m=1}^{\infty} e^{-2 m \sqrt{\pi s}}+11 \frac{\sqrt{\pi}}{\sqrt{s}} .
\end{aligned}
$$

\section{ACKNOWLEDGMENT}

We wish to thank Floyd L. Williams, University of Massachusetts at Amherst, and the editor for their kindness in providing suggestions which proved to be most helpful.

\section{REFERENCES}

1. Bellman, R., A Brief Introduction to Theta Functions, Holt, Rinehart \& Winston, NY, 1961, p. 24. MR 23:A2556

2. Beyer, William H. (editor), CRC Standard Mathematical Tables, 26th ed., CRC Press, Inc., Boca Raton, Florida, 1981, p. 398.

3. Erdelyi, A. (editor), Tables of Integral Transforms, Vol. I, Bateman Manuscript Project, McGraw-Hill Book Co., Inc., NY, 1954, pp. 133, 137, 143-144, 146. MR 15:868a

4. Gradshetyn, I. S. and Ryzhik, I. M., Tables of Integrals, Series and Products, Academic Press, NY, 1965, p. 967. MR 33:5952

5. Keating, S. F., An infinite family of zeta functions indexed by Hermite polynomials, Journal of Mathematical Analysis and Applications, Vol. 140, No. 1 (1989), pp. 218-223. MR 91b:11092

6. Lebedev, N. N., Special Functions and Their Applications, Dover Publications, Inc., NY, 1972, p. 60. MR 50:2568

7. Marklof, J., Theta sums, Eisenstein Series, and the semiclassical dynamics of a precessing spin, in Emerging Applications of Number Theory (D. A. Hejhal et al., eds.), IMA Vol. 109, Springer-Verlag, 1999, pp. 405-450. MR 2000k:81094

8. Ogg, Andrew, Modular Forms and Dirichlet Series, W. A. Benjamin, Inc., NY, 1969, pp. x, xi. MR 41:1648

9. Sarnak, Peter, Some Applications of Modular Forms, Cambridge University Press, NY, 1990, pp. 10-11. MR 92k:11045 
10. Shimura, G., On the transformation formulas of theta series, Amer. J. Math., Vol. 115 (1993), pp. 1011-1052. MR 94h:11045

11. Vigneras, Marie-France, Séries theta des formes quadratiques indéfinies, Lecture Notes in Mathematics 627, Springer-Verlag, NY, 1977, pp. 227-239. MR 58:521

Department of Mathematics and Computer Science, Eastern Connecticut State University, Willimantic, Connecticut 06226

E-mail address: keating@easternct.edu 